Z. Klin. Chem. Klin. Biochem.

12. Jg. 1974 , S. $276-278$

\title{
Eine mechanisierte kompetitive Proteinbindungsanalyse für Cortisol im Serum ohne vorherige Extraktion mit organischen Lösungsmitteln ${ }^{1}$ )
}

\author{
Von O. A. Müller, J. Braun, Renate Fröhlich und P. C. Scriba \\ Aus der II. Medizinischen Klinik (Direktor: Prof. Dr. E. Buchborn) der Universität München
}

(Eingegangen am 9. November 1973/18. Februar 1974)

\begin{abstract}
Es wird eine kompetitive Proteinbindungsanalyse für Serum-Cortisol beschrieben, welche erstmals erlaubt, ohne Extraktion mit organischen Lösungsmitteln zu arbeiten. Dabei werden auf derselben Dextran-Gel-Säule die Freisetzung des Cortisols aus der Proteinbindung durch Ansäuerung, die Reaktion mit an Cortisol bindendem globulinreichem Serum und die B/F-Trennung durchgeführt. Durch Mechanisierung (simultane Säulenchromatographie) können gleichzeitig 25 Proben (reine Chromatographiezeit: 48 Minuten) bestimmt werden. Die Richtigkeit, Spezifität und Präzision der Methode wurden untersucht und sind für diagnostische Zwecke voll befriedigend. Die Werte von Normalpersonen $(n=68)$ für das $9^{\circ}{ }^{\circ}$-Nüchtern-Cortisol liegen zwischen 45 und $200 \mu \mathrm{g} / 1$. Die Normalwerte für die Stimulation mit ACTH und die Suppression mit Dexamethason werden angegeben. Das beschriebene Prinzip eignet sich auch für die radioimmunologische Cortisolbestimmung.
\end{abstract}

\section{A mechanized competitive protein binding analysis for serum cortisol which avoids extraction with organic solvents}

A modification of competitive protein binding analy sis for serum cortisol is described, which avoids the extraction with organic solvents necessary in other procedures: The same dextran gel column liberates cortisol from its binding proteins in an acidification step, then serves as the site for the reaction of a cortisol binding globulin-rich-serum with the cortisol and is finally used for the separation of bound and free hormone. The whole chromatographic procedure lasts 48 minutes. The mechanization (simultaneous column chromatography) enables us to measure 25 samples at a time. Recovery, specificity and precision of the method are sufficient for diagnostic purposes. The serum cortisol level at $9.00 \mathrm{a}$. m. in 68 normal subjects ranged from 45 to $200 \mathrm{\mu g} / \mathrm{l}$. The normal responses to ACTHstimulation and dexamethasone suppression were evaluated. The principle of this procedure is also suitable for cortisol radioimmunoassay.

Die auf den Arbeiten von Murphy (1) basierenden kompetitiven Proteinbindungsanalysen für Steroide, insbesondere für Cortisol, erfordern alle eine vorherige Extraktion mit organischen Lösungsmitteln (2). Eine Mechanisierung einer kompetitiven Proteinbindungsanalyse für Cortisol ist allein schon wegen dieser Extraktion mit großen technischen Schwierigkeiten verbunden. Es wird hier eine kompetitive Proteinbindungsanalyse beschrieben, bei der erstmals die Extraktion mit organischen Lösungsmitteln vermieden werden kann. Vielmehr werden die drei wesentlichen Schritte einer kompetitiven Proteinbindungsanalyse auf derselben Dextran-GelSäule durchgefuihrt: 1. die Freisetzung des Cortisols aus der Proteinbindung, 2. die Reaktion mit Transcortin-reichem Serum und 3. die Trennung der Transcortin-gebundenen von der nicht gebundenen Steroidfraktion.

\section{Methodik}

$\mathrm{Zu} 100 \mu \mathrm{l}$ Serum bzw. Standard werden $100 \mu \mathrm{l}$ eines ${ }^{3} \mathrm{H}$-Cortisol-Arbeitsstandards (1:2000-Verdünnung eines ${ }^{3} \mathrm{H}$-CortisolTracers mit einer spezifischen Aktivität von $30 \mathrm{Ci} / \mathrm{mmol}$, Radiochemical Center, Amersham) und $100 \mu 10,1 \mathrm{~mol} / 1 \mathrm{HCl}$ pipettiert. Dieses Gemisch wird auf eine temperaturkonstante $\left(26^{\circ} \mathrm{C}\right)$, angesäuerte $(0,1 \mathrm{~mol} / 1 \mathrm{HCl}, \mathrm{pH} 1,0)$ Sephadex G-10Säule (2 g Sephadex G-10, Fa. Pharmacia, Uppsala, im Zylinder einer 5 ml-Einmalspritze, Fa. Braun, Melsungen) gepumpt (Mikropumpe, Fa. Ismatec, Zürich). Mit $0,2 \mathrm{~mol} / 1$ Tris= $\mathrm{HCl}-$ Puffer, $\mathrm{pH} 8,0$, werden die Serumproteine eluiert und verworfen, während Cortisol - z. T. auch andere Steroide - quantitativ am Gel adsorbiert bleibt. Auf der jetzt umgepufferten Säule erfolgt anschließend die kompetitive Proteinbindungsreaktion mit $0,5 \mathrm{ml}$ eines verdünnten (ca. 1:50) Transcortinreichen Serums (Östrogen-behandelte Patienten nach Dexamethason-Gabe (3)). Der Transcortin-gebundene ${ }^{3} \mathrm{H}$-CortisolAnteil (= B) wird wiederum mit 0,2 mol/1 Tris-HCl-Puffer,

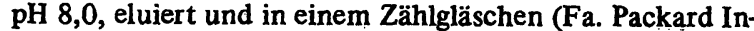
strument, Frankfurt) aufgefangen. Nach Ansaugen von $1,2 \mathrm{ml}$ eines Nachwaschserums (gepooltes, mit Florisil ausgeschütteltes, 1:3 verdünntes Serum aus den Serumresten unseres Laboratoriums für Klinische Chemie) wird der auf der Säule verbliebene, ${ }^{3} \mathrm{H}$-Cortisol-Anteil (freier Hormonanteil $=\mathrm{F}$ ) durch Elution mit $0,1 \mathrm{~mol} / 1 \mathrm{HCl}$ quantitativ in einem zweiten Zählgläschen gesammelt. Auf derselben Säule kann damit sofort die nächste Analyse durchgeführt werden. Die reine Chromatographiezeit beträgt 48 Minuten (u. a. abhängig vom Schlauchsystem). Abbildung 1 zeigt ein Elutionsdiagramm unter dieșen Bedingungen. Gezählt werden die Aktivitäten von B und F (Zählgläschen 1 und 2) nach Zugabe eines Szintillationsgemisches (Insta-Gel, Fa. Packard Instrument, Frankfurt). B (oder F) wird in Prozent von der jeweiligen Gesamtaktivität $(=\mathrm{B}+\mathrm{F})$ - ohne Quench-Korrektur - errechnet (Olivetti Tischcomputer $P$ 102). Dieses Vorgehen hat sich wegen Schwankungen der Gesamt-Aktivität (= B + F) um etwa 10 bis. $15 \%$ bewährt. Hauptursache dieser Schwankungen ist ein z. T. unterschiedlicher Quench bei der $\beta$-Szintillationszählung, der vor allen

1) Mit Unterstützung der Deutšchen Forschungsgemeinschaft (SFB 51) 


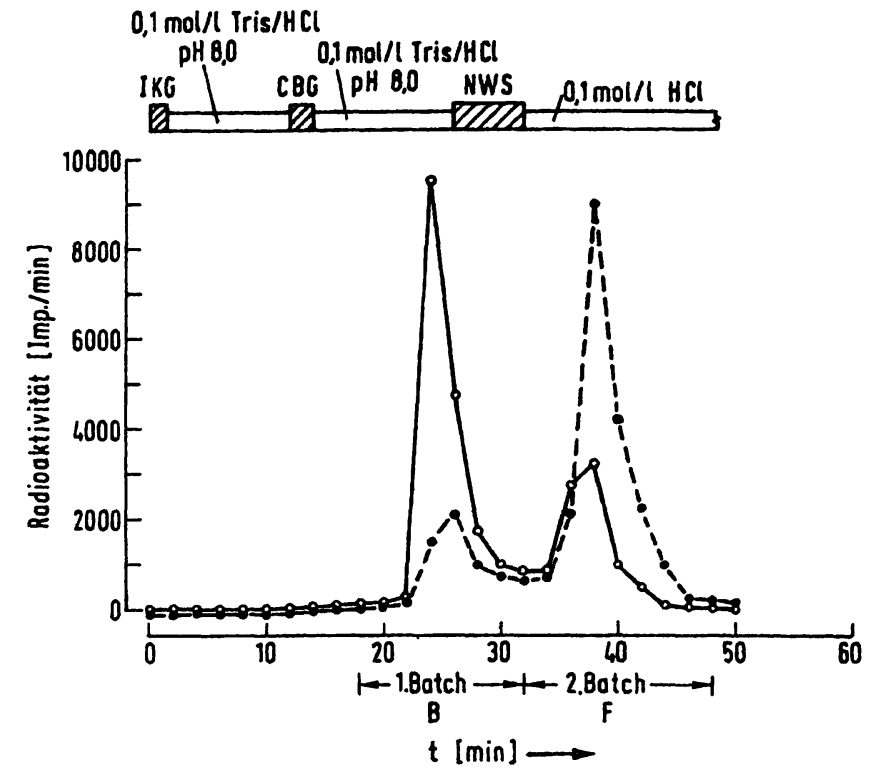

Abb. 1. Elutionsdiagramm: Extraktion, Transcortin-Bindung und die B/F-Trennung von Cortisol auf Sephadex G-10

Flußrate pro min: etwa $0,2 \mathrm{ml}$.

Aufgezeichnet sind die Elutionsprofile eines Leerwertes

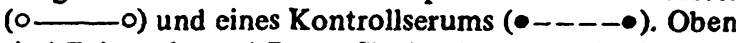
sind Zeitpunkt und Dauer für das Ansaugen des Inkubationsgemisches (IKG), des Puffers, des Transcortin-reichen Serums (CBG), des Nachwaschserums (NWS) und der Säure angegeben. Unten sind die von uns gewählten Zeiten für das Sammeln vom 1 . Batch $(=B=$ der durch CBG eluierte Anteil) und vom 2. Batch (=F $=$ der durch NWS eluierte Anteil) aufgefürt. Die zeitliche Verschiebung zwischen Ansaugen z. B. des Transcortin-Serums und der Elution des 1. Batch ist durch die Wegstrecke (Schlauchsystem, Pumpe und Sephadex-Säule) zwischen Ansaugen und Sammeln bedingt. Das Tal zwischen beiden Batch-Gipfeln fält nicht ganz mit der Null-Linie zusammen, weil das auf dem Sephadex G-10 extrahierte Cortisol im geringen Maße und nahezu konzentrationsunabhängig von der Säule spontan, d. h. nur mit Puffer, eluiert wird.

Dingen durch geringe Unterschiede der Batch-Volumina entsteht. Eine Quench-Korrektur erwies sich als sehr aufwendig (interner Standard) oder fehlerhaft (externer Standard). \% B (oder \% F) werden gegen die Cortisol-Standards aufgetragen und die Serumwerte an dieser Eichkurve abgelesen. Neuerdings erfolgt die Auswertung mittels einer EDV-Anlage nach einem Fortran-Programm auf der Grundlage der Spline Approximation (4).

Bei der Mechanisierung konnte auf die Erfahrungen mit der mechanisierten simultanen Säulenchromatographie beim $T_{3}$-in vitro-Test (5) und bei der Gesamt- $T_{4}$-Bestimmung (6) zurückgegriffen werden. Mittels eines Chromatographiegerätes (Fa. Sartorius, Göttingen, in Vorbereitung) können in einem Durchgang gleichzeitig 25 Bestimmungen (25 Säulen) in einem geschlossenen, temperaturkonstanten System durchgeführt werden. Utber Einzelheiten des Gerätes und die darauf durchführbaren Hormonbestimmungen wird gesondert berichtet werden (in Vorbereitung).

\section{Ergebnisse und Diskussion}

Abbildung 2 zeigt eine typische Eichkurve. Die Methode ist empfindlicher zu machen (z. B. Temperatur, Tracerund Transcortin-Serum-Verdünnung), es kam uns aber auf einen für die Routine zweckmäßigen Meßbereich an.

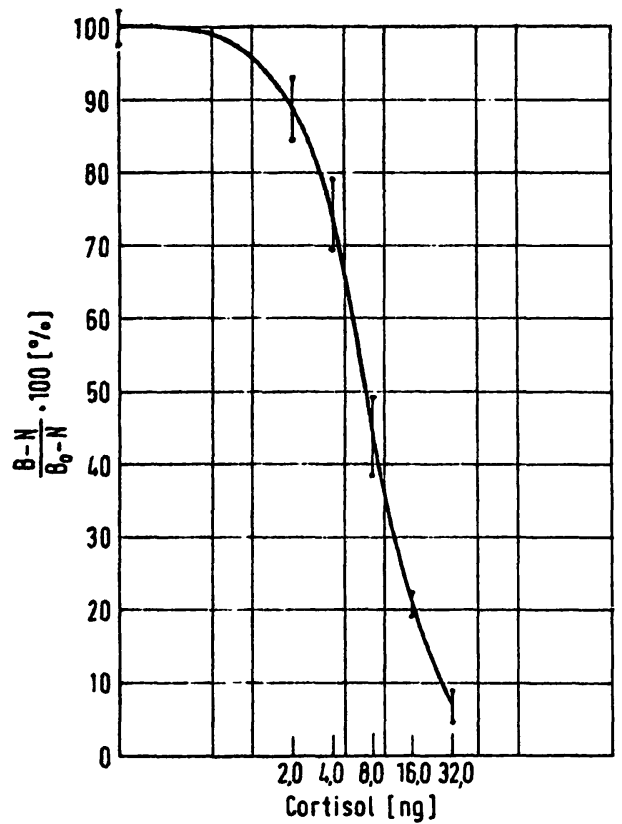

Abb. 2. Cortisol-Standardkunve

Berechnet und gezeichnet mittels einer Siemens 404/3 Datenverarbeitungsanlage und einem Hagen Graphomaten nach einem FORTRAN-Programm auf der Grundlage der Spline-Approximation (4).

Ordinate: $\frac{\mathrm{B}-\mathrm{N}}{\mathrm{B}_{0}-\mathrm{N}} \cdot 100[\%]$, linear

Abszisse: ng Cortisol pro Ansatz, logarithmisch.

Die Doppelwerte sind angegeben.

Dieser liegt hier bei etwa 0-20 ng Cortisol im Ansatz, entsprechend 0-200 $\dot{\mu} \mathrm{g} / \mathrm{l}$. Bei höheren Werten, z. B. nach ACTH, werden $100 \mu$ leines 1:1 bzw. 1:2 oder $1: 3$ verdünnten (dest. Wasser) Serums eingesetzt. Die Empfindlichkeit (3s-Bereich des Null-Wertes) liegt unter diesen Bedingungen - etwas schwankend von Versuch zu Versuch - bei 1 bzw. 2 ng Cortisol im Ansatz, entsprechend $10-20 \mu \mathrm{g} / 1$.

Die Präzision in der Serie (Serum) ergab mit einem Variationskoeffizienten (VK) von $9,1 \%(75 \pm 6,9 \mu \mathrm{g} / 1$ Cortisol $(\bar{x} \pm s, n=21)$ ebenso wie die Qualitätskontrolle von Tag zu Tag mit einem VK von 12,1\% (gepooltes Kontrollserum: $142 \pm 17 \mu \mathrm{g} / \mathrm{l}$ Cortisol $(\overline{\mathrm{x}} \pm \mathrm{s}), \mathrm{n}=$ 46) zufriedenstellende Werte. Die Streuung der Werte im untersten Meßbereich (z. B. Serum-Cortisolspiegel nach Dexamethason) ist naturgemäß größer (z. B. $8,9 \pm 5,8 \mu \mathrm{g} / 1, \overline{\mathrm{x}} \pm \mathrm{s}, \mathrm{n}=24$ ), ohne daß damit die diagnostische Wertigkeit eingeschränkt ist.

Die Wiederfinderaten von Cortisol-Standards $(1,2,3$, $5,10 \mathrm{ng}$ im Ansatz) im Serum lagen bei $96 \pm 5 \%$ $(\bar{x} \pm s, n=20)$. Die Werte für verschiedene Verdünnungen eines Serums lagen auf der Eichkurve.

Es wurde von uns die Mitreaktion anderer Steroidhormone bei einer kompetitiven Proteinbindungsanalyse nach Dichlormethan-Extraktion (7) und der hier geschilderten Methode auf Sephadex G-10 ohne vorherige Extraktion mit organischen Lösungsmitteln verglichen (Abb. 3). Dabei wurden entsprechend dem Vorgehen 


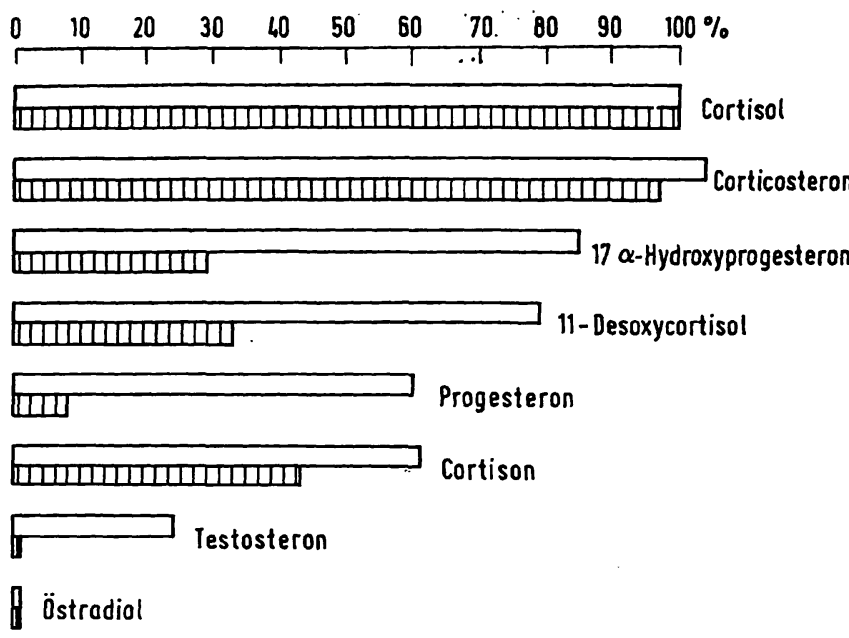

Abb. 3. Mitreaktion anderer Steroidhormone (in \% Cortisol) bei der kompetitiven Proteinbindungsanalyse für Cortisol:

$\square \begin{aligned} & \text { kompetitive Proteinbindungsanalyse nach } \\ & \text { Dichlormethan-Extraktion }\end{aligned}$
ШणD] $\begin{aligned} & \text { kompetitive Proteinbindungsanalyse auf Sepha- } \\ & \text { dex G-10-Säulen }\end{aligned}$
Einzelheiten im Text

mit den Cortisol-Standards andere Steroide (verschiedene Konzentrationen) eingesetzt und ihre Mitreaktion in \% Cortisol aus den Versuchsergebnissen errechnet. Die Werte nach Dichlormethan-Extraktion entsprechen in etwa den Angaben aus der Literatur, wobei Unterschiede vor allen Dingen auf die verschiedenen $\mathrm{B} / \mathrm{F}$ Trennsysteme -- bei uns Sephadex G-50 superfine (7) zurickzufuihren sind (1). Die deutlich geringere Mitreaktion von Progesteron, 17 $\alpha$-OH-Progesteron, Testosteron und 11-Desoxycortisol in der hier geschilderten kompetitiven Proteinbindungsanalyse (ohne vorherige Extraktion mit organischen Lösungsmitteln) im Vergleich zur kompetitiven Proteinbindungsanalyse nach Dichlormethan-Extraktion beruht auf einer stärkeren, wenn auch reversiblen Bindung dieser Hormone an Sephadex G-10. Auch unter Berücksichtigung eventuell sehr hoher Progesteron-Spiegel (z. B. im 3. Trimenon) sind die Säulen allerdings immer wieder zu verwenden, da durch das Nachwaschserum und das Spülen mit $\mathrm{HCl}$ das Progesteron eluiert wird und zu keiner Verfälschung der folgenden Analyse führt (7). Die quantitativ günstigeren Spezifitätsergebnisse dieser kompetitiven Protein- bindungsanalyse ändern aber nichts an der prinzipiellen Einschränkung der Spezifität einer kompetitiven Proteinbindungsanalyse für Cortisol. Diese ist durch die mehr oder weniger starke Bindungsaffinität auch von anderen Steroiden zum Transcortin bzw. zu den im Transcortin-Serum noch vorhandenen anderen SerumProteinen bedingt. Allerdings beeinträchtigt das die Richtigkeit der Cortisolwerte und damit den Einsatz einer solchen Methode bei klinischen Routine-Messungen praktisch nicht, da die Serumspiegel der mitreagierenden Steroide - sieht man von Schwangeren, Neugeborenen und Patienten mit adrenogenitalem. Syndrom $\mathrm{ab}-\mathrm{sehr}$ viel niedriger als die Cortisolspiegel sind (8).

Es wurden mit dieser Methode folgende Normalwerte ermittelt:

Die 9 Uhr-Nüchtern-Cortisolwerte von 68 Normalpersonen (endokrin gesunde Patienten und Klinikpersonal) lagen im Mittel bei $111 \pm 37 \mu \mathrm{g} / 1(\overline{\mathrm{x}} \pm \mathrm{s})$, der höchste Wert bei $200 \mu \mathrm{g} / 1$, der niedrigste bei $45 \mu \mathrm{g} / 1$. Nach einer vierstïndigen ACTH-Infusion (0,25 mg Synacthen) fand sich ein Cortisol-Anstieg von $136 \pm$ $36 \mu \mathrm{g} / 1$ auf $328 \pm 71 \mu \mathrm{g} / 1(\overline{\mathrm{x}} \pm \mathrm{s}, \mathrm{n}=19)$. Bei den gleichen Patienten fand sich 30 Minuten nach einer raschen i. v. Injektion von $0,25 \mathrm{mg}$ Synacthen ein praktisch gleicher Cortisol-Anstieg von $134 \pm 36 \mu \mathrm{g} / 1$ auf $296 \pm 82 \mu \mathrm{g} / 1(\overline{\mathrm{x}} \pm \mathrm{s}, \mathrm{n}=19)$. Elf Stunden nach Suppression (9 Uhr früh) mit einer einmaligen oralen Gabe von $2 \mathrm{mg}$ Dexamethason (22 Uhr) ergab sich ein mittlerer Cortisolspiegel von $26 \pm 8 \mu \mathrm{g} / \mathrm{i}(\mathrm{x} \pm \mathrm{s}, \mathrm{n}=14)$. Damit war dieser Bereich von der untereñ Normalbereichsgrenze abgesetzt. Diese Resultate stimmen gut mit den von anderen Autoren mit vergleichbaren Methoden gewonnenen Ergebnissen überein (Übersicht bei 1. c. 9).

Der hauptsächliche Vorteil unserer Methode liegt in der Vermeidung der Extraktion mit organischen Lösungsmitteln und der damit möglichen Mechanisierung. Eine Erprobung dieses Verfahrens für andere Körperflüssigkeiten, z. B. Urin, steht noch aus. Dagegen ist die Anwendung dieses Methodenprinzips auf die radioimmunologische Serumcortisol-Bestimmung, als Mod:fikation also lediglich Einsatz eines Cortisol-Antikörpers statt des Transcortin-Serums, nach den ersten Ergebnịssen gut möglich.

\section{Literatur} 1. Murphy, B. E. P. (1967), J. Clin. Endocrinol. Metab. 27,

2. Breuer, H. (1973), diese Z. 11, 357-361. 3. Johansson, E. D. B. (1969), Acta Endocrinol. (Copenhagen)
$61,592-606$.

4. Marschner, I., Erhardt, F. \& Scriba, P. C. (1973), IAEASymposium, Istanbul, in press. 5. Horn, K., Henner, J. \& Scriba, P. C. (1971), Mitt. Deut. Ges.
Klin. Chem. 2, 29-33.

6. Horn, K., Habermann, J., Henner, J., zur Horst, I. \& Scriba, P. C. (1972), Z. Anal. Chem. 259, 222, Abstract.

7. Braun, J. (1973), Inauguraldissertation, München.

8. Pirke, K. M. \& Stamm, D. (1972), diese Z. 10, 254-259.

9. Newsome, H. H., Clements, A. S. \& Borum, E. H. (1972) J. Clin. Endocrinol. Metab. 34, 473 -483 .
Prof. Dr. P. C. Scriba

II. Med. Klinik d. Univ. 8 München 2

Ziemssenstraße 1 